\title{
Sleep in Tourette Syndrome
}

\author{
Roumen Kirov • Andreas Becker • Aribert Rothenberger
}

Published online: 4 September 2014

(C) Springer International Publishing Switzerland 2014

\begin{abstract}
Tourette syndrome (TS) is a heritable developmental neuropsychiatric disorder and is defined as part of a spectrum of motor and vocal tic disorders (TD). Sleep problems are a common feature associated with TS/TD. They may affect daytime tic expression and neurobehavioral functioning. However, sleep studies on TS/TD are still scarce, and firm conclusions about the relationship of tics and sleep are difficult to draw. In this review, we summarize the existing knowledge about sleep problems in TS/TD and advocate for better understanding of their nature. Neglected links between the neurobiology of sleep and the psychophysiology of TS/TD and their psychiatric comorbidity, as well as medication for $\mathrm{TS} / \mathrm{TD}$, are highlighted, and future trends in sleep research on TS/TD are proposed.
\end{abstract}

Keywords Sleep - Tourette syndrome and other tic disorders . Comorbidity $\cdot$ Medication $\cdot$ Neurobiology $\cdot$ Future research

\section{Introduction}

Tourette syndrome (TS) is a neuropsychiatric disorder and is defined as part of a spectrum of tic disorders (TD), with the TD

\footnotetext{
R. Kirov

Institute of Neurobiology, Bulgarian Academy of Sciences, Acad. G. Bonchev Str.23, 1113 Sofia, Bulgaria

e-mail: ru@bio.bas.bg

e-mail: roumen_kirov@yahoo.com

A. Becker $\cdot$ A. Rothenberger $(\square)$

Clinic for Child and Adolescent Psychiatry, University Medical Center Göttingen, von Siebold-Strasse 5, 37075 Göttingen, Germany e-mail: arothen@gwdg.de

A. Becker

e-mail: abecker4@gwdg.de

being subdivided and classified into persistent or chronic motor and/or vocal TD (lasting more than 1 year), and provisional or transient TD (lasting less than 1 year) [1-4]. TD are heritable and frequent $(\sim 1 \%$ for TS and $\sim 5 \%$ for other chronic TD) developmental neuropsychiatric disorders worldwide, with early childhood onset (at age 4-6 years) or prepubertal onset (at age $>8$ or $<18$ years) and with a male-to-female ratio ranging from 3:1 to 4.3:1 [2-5]. TD including TS are clinically characterized by sudden, rapid, and repetitive but nonrhythmic motor movements or vocalizations called tics, usually appearing in bouts, while waxing and waning in frequency, intensity, localization, and typology $[3,4,6]$. In children older than 10 years, tics are usually preceded by premonitory sensorimotor phenomena, including irresistible urges to move, and may also be triggered by psychosocial stress, anxiety, emotional excitement, or fatigue $[3,4,6]$. Intriguingly, tics can be suppressed transiently by activities that require focused attention and/or attention allocation and executive and fine motor control, such as task performance, reading aloud, playing a musical instrument, or engaging in certain sports $[3,4,6]$. Tic severity usually peaks during the second decade of life ( $\sim 10.6$ years), with most patients showing a marked reduction in tic severity by the end of adolescence $[4,6]$. Only $20 \%$ of children or fewer with TS/ TD continue to experience a moderate level of impairment of global functioning by the age of 20 years. However, in approximately one third of patients with TS/TD, tics continue to persist into adulthood and can be associated with the most severe symptoms, including violent episodes of self-injurious motor tics or socially stigmatizing coprolalic utterances or gestures $[4,6]$. Thus, daytime symptom expression in TS/TD is characterized by extremely high intra- and interindividual variability in the course of the disorder and across the lifespan $[4,6]$. Notably, one important aspect of TS/TD phenomenology is the fact that tics may also persist during sleep [7-9].

Epidemiologically, TS/TD are signified by a high prevalence rate of psychiatric comorbidities [4, 6, 10-12], which 
may additionally worsen tic expression and psychosocial functioning in TS/TD patients and thus may produce sleep alterations that are different from the presence of tics during sleep [7-9]. For instance, the prevalence rate of attentiondeficit/hyperactivity disorder (ADHD) and obsessive-compulsive disorder (OCD) comorbidities in TS/TD is up to 50$70 \%$, and up to $10 \%$ of patients with TS/TD have coexistent depression, anxiety, and self-injurious behavior [4, 6, 10-12]. Given the high prevalence of psychiatric comorbidities in TS/ TD patients and the recent progress in understanding the phenomenology, etiology, and neurobiology of these neuropsychiatric conditions, it has recently become clear that TS/ TD represent a significant health problem worldwide, which can lead to impairments in patients' social competence and in their academic and occupational achievements, and is likely to cause difficulties in their lives and in the lives of their relatives $[13 \bullet \bullet]$.

The etiology of TS/TD is complex and is still elusive. Their genetic background might be related to deviations in single nucleotide polymorphisms in genes regulating dopamine, noradrenaline, and serotonin (5-hydroxytryptamine [5-HT]) metabolism, with these genetic mechanisms being proposed to have much in common with those proposed for ADHD and OCD $[4,6,14]$. Further, gene-environment interaction and postinfection autoimmune processes have also been suggested to play a role in TS/TD etiology [6].

\section{Pathophysiology of TS/TD}

Pharmacological studies in TS/TD have indicated dopamine overactivity in the striatum and striato-cortical circuits, elevated noradrenaline ascending modulation influences on the cortex, and decreased 5-HT neuromodulation [6, 15]. Transcranial magnetic stimulation (TMS) studies have shown motor loops and intracortical and cortico-subcortical hyperactivation in these patients [16]. On the basis of electroencephalogram (EEG) studies and the related deviant oscillatory patterns that have been found, the following hypotheses for the appearance of tics have been proposed: (1) loss of basal ganglia control; (2) thalamocortical dysrhythmia (replacing normal thalamocortical oscillations with low-frequency rhythms in the theta range), which transmits the misguided basal ganglia signals to the cortex; and (3) frontal lobe compensation originating from the prefrontal cortex (PFC), which adaptively modulates the misguided striatal and thalamocortical oscillations that are characteristic of TS/TD. Repeated activation of these frontal systems can lead not only to tic suppression but also to willful alteration of the character of the movements involved [17]. Functional neuroimaging data have confirmed these hypotheses by demonstrating aberrant activity in the basal ganglia and abnormal basal ganglia-cortex interplay through impaired cortico-striato-thalamocortical loops, paralleled by deficient limbic cortico-subcortical loops, with the brain areas involved in executive control being preserved [18•]. Yet no data concerning brain activation patterns during sleep in patients with TS/TD so far exist.

\section{Pharmacological Treatment of TS/TD}

Worldwide, there are several substances in clinical use, but no standard medication therapy for TS/TD exists, because the evidence base for these drugs is scarce and thus pharmacological treatment for TS/TD is recommended only in cases with psychosocial impairment by moderate to severe tics and clinically significant psychiatric comorbidities in TS/TD - in which cases, social interaction is hampered and is accompanied by substantial difficulties in the patient's life and in the life of their parents and relatives $[19,20]$. The first drugs used to suppress tics have been dopamine-2 receptor antagonists [6]. Depending on tic severity, the related stress and emotional problems, and the type of psychiatric comorbidities, the currently used drugs for treatment of TS/TD include typical neuroleptics (e.g., haloperidol), antinoradrenergic drugs (e.g., clonidine), atypical neuroleptics (e.g., risperidone and aripiprazole), and benzamides (e.g., tiapride and sulpiride). Antidepressants such as selective serotonin reuptake inhibitors (e.g., fluvoxamine), dopamine and noradrenaline agonists (e.g., methylphenidate and atomoxetine), and their combinations might come into play if comorbidities have to be treated in parallel $[19,20]$. Notably, all of these drugs can significantly affect sleep regulatory mechanisms and produce various sleep alterations [21-23]. Thus, along with tic severity and related stressful and emotional problems and psychiatric comorbidities, medication appears to be an additional factor either in improving sleep or in leading to disturbed and altered sleep in patients with TS/TD, depending on the psychopathological situation.

\section{Neurobiology of Sleep}

Sleep is a universal state in all organisms, which serves vitally important brain and body homeostatic functions and a range of cognitive processes, including memory consolidation and transformation, and emotional processing and regulation [24-28]. Insufficient and disturbed sleep results in learning problems, inattention, diminished executive control, and a range of stressful and emotional problems [21, 28].

Sleep Phenomenology

Human sleep architecture can be objectively evaluated by polysomnographic records, which traditionally use electrophysiological techniques, including EEG, electromyography, 
and detection of eye movements to characterize sleep at system levels. This polysomnographic method is called polysomnography (PSG) and is considered as "the gold standard" in sleep research because it can reliably document brain activation patterns and important body arousal and homeostatic indicators [29, 30]. For clinical purposes, routine PSG can be supplemented by surface electromyography from the anterior tibial muscles to detect sleep disorders such as periodic limb movements in sleep (PLMS) and restless legs syndrome (RLS), as well as by recordings of cardiorespiratory events in sleep, such as sleep-disordered breathing [30]. In humans, overnight sleep is characterized by a cyclic occurrence of non-rapid eye movement (NREM) sleep and rapid eye movement (REM) sleep. NREM sleep includes lighter sleep stages 1 and 2, and stages 3 and 4 of the deeper slowwave sleep (SWS) [29, 30], with stages 3 and 4 also being recognized as N3 sleep, according to the Manual for the Scoring of Sleep and Associated Events, published on behalf of the American Academy of Sleep Medicine in 2007 [30]. Whereas SWS dominates the first half of the night, REM sleep and stage 2 of NREM sleep dominate the second half. Normally, sleep onset begins with a brief period of stage 1 of NREM sleep, which is subsequently followed by sleep deepening, marked by the appearance of stage 2 of NREM sleep and a further progressive transition to stages 3 and 4 of SWS. The latter is followed by a relatively short, transient stage 2 of NREM sleep, after which a period of REM sleep appears. This progression of sleep stages and, in particular, the NREM sleep-REM sleep alternation, forms one sleep cycle of approximately $90 \mathrm{~min}$ duration. About five such sleep cycles are usually observed in normal human overnight sleep [29, 30].

Importantly, to reliably characterize various types of movements in sleep, including tics, all methods of sleep evaluation (PSG, actigraphy, and subjective self- or observer-reported questionnaires and diaries) require videomonitoring [30].

\section{Sleep-Wake Regulation}

As shown in Fig. 1. (upper panel), two major, anatomically and neurochemically distinct brain structures are generally involved in sleep-wake regulation. The first one engages hypothalamic nuclei located in the diencephalon (the suprachiasmatic nucleus and lateral hypothalamic nuclei), regulating the sleep-wake switch, while the second one engages nuclei located in the mesopontine brainstem, regulating NREM-REM sleep alternation [31]. In brief, circadian clock genes located in the suprachiasmatic nucleus transmit sleepinitiating signals not only to the mesopontine brainstem nuclei but also to all neuronal populations in the brain and body. Also, they act to release the sleep-inducing "hormone" melatonin from the pineal gland [31]. In parallel, orexin/ hypocretin-containing neurons located in the lateral hypothalamus (which - along with their important role in sleep-wake regulation - govern sexual, eating, and locomotor behaviors and reward [32]) transmit neurochemical signals to the dopaminergic ventral tegmental area and nucleus accumbens [33] - dopaminergic brain structures that exert strong modulation on the mesopontine brainstem nuclei responsible for the regulation of NREM-REM sleep alternation in the course of sleep [34] (Fig. 1. upper panel).

The mesopontine brainstem system consists of REM-off (silent during REM sleep) noradrenaline (located in the locus coeruleus) and 5-HT (located in the raphe nuclei) neurons and REM-on (active during REM sleep) acetylcholine (located in the pedunculopontine tegmental nucleus and laterodorsal tegmental nucleus) and glutamate/aspartate (located in the brainstem reticular formation) neurons. These REM-off/ REM-on neurons act reciprocally in the course of sleep through a complex glutamate and gamma-amino-butyric acid neural complex [31]. Importantly, this reciprocal interaction mesopontine brainstem system projects monoaminergic/ acetylcholinergic influences to the thalamocortical neurons and is responsible for the dramatic changes in brain oscillations and activation patterns across distinct sleep-wake stages [31] (Fig. 1. upper panel).

Neglected Links between the Phenomenology and Pathophysiology of TS/TD and Sleep Regulatory Mechanisms

1. Clinically, since daytime symptom expression in TS/TD is characterized by high intra- and interindividual variability $[3,4,6,17]$, it could be expected that sleep disturbances and alterations in these patients depend strongly on tic severity, the waxing and waning course, and the symptom profile. However, only two PSG studies so far have indicated such a link indirectly by demonstrating a positive correlation between sleep alterations and tic severity $[35$, 36]. An influence in both directions cannot be excluded.

2. It should be emphasized that the sleep-wake pattern and its regulatory mechanisms mature from infancy to childhood and further through childhood and adolescence. These developmental changes are mostly marked by a progressive decrease in REM sleep from infancy to childhood, and from childhood to late adolescence [37]. More recent studies have shown a decrease and topographic changes of NREM sleep EEG slow $(\sim 1-4 \mathrm{~Hz})$ oscillations, paralleled by a decrease in REM sleep theta $(\sim 5-$ $7 \mathrm{~Hz}$ ) EEG power from childhood to adolescence, with gender differences also being found [38, 39]. These developmental changes in sleep architecture and sleep EEG signatures are recognized as reflecting synaptic pruning and maturation of brain networks [21, 22, 38, 39]. This issue mandates particular attention to a broad spectrum of developmental neuropsychiatric conditions, since even subtle sleep alterations during this period may produce 
Fig. 1 Multifactorial model of sleep disturbances in Tourette syndrome and tic disorders. 5- $H T$ 5-hydroxytryptamine (serotonin), Ach acetylcholine, As aspartate, $B R F$ brainstem reticular formation, $G l u$ glutamate, $L C$ locus coeruleus, $L T D$ laterodorsal tegmental nucleus, $N A$ noradrenaline, $N A c c$ nucleus accumbens, NREM non-rapid eye movement sleep, $P P T$ pedunculopontine tegmental nucleus, $R E M$ rapid eye movement sleep, $R N$ raphe nuclei, $V T A$ ventral tegmental area. (Upper panel based on PaceSchott and Hobson [31], Li et al. [32], Hrabovszky et al. [33], and Monti and Monti [34]; lower panel based on Roessner et al. [4], Swain et al. [6], Rothenberger et al. [9], Rothenberger and Roessner [10], Ferao et al. [11], Moll et al. [16], Leckman et al. [17], Ganos et al. [18•], Brand and Kirov [21], and Kirov and Brand [22])

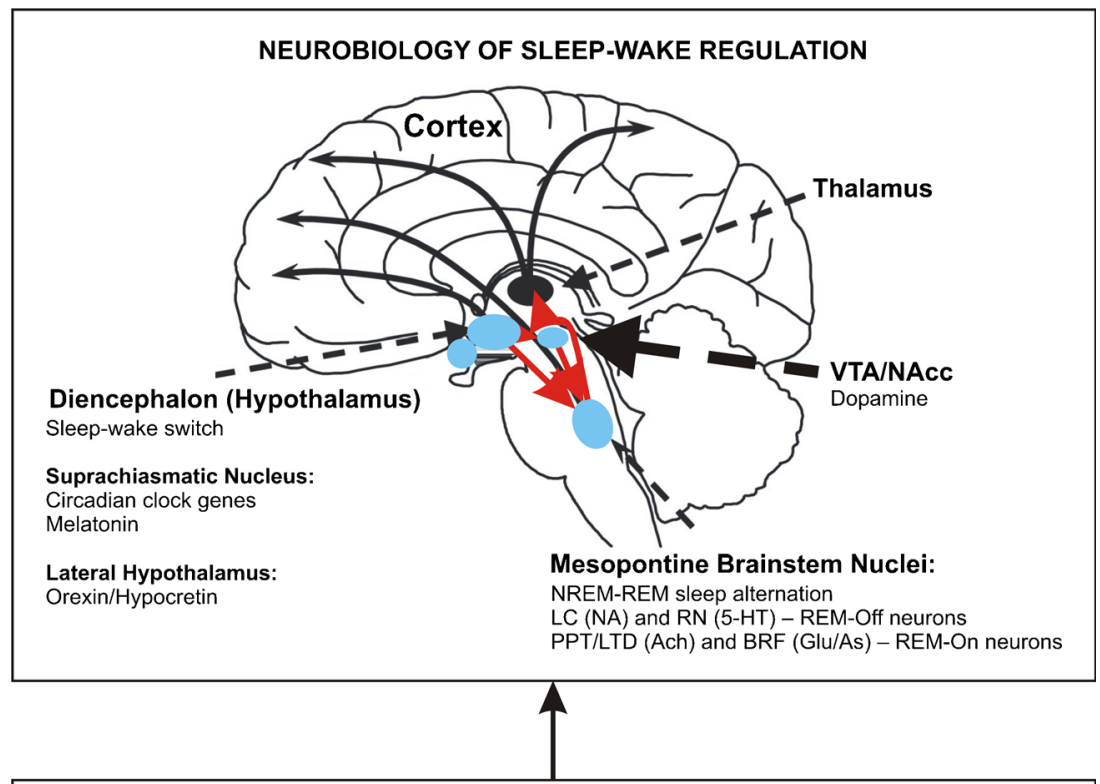

FACTORS THAT COULD AFFECT THE SLEEP-WAKE REGULATION

1. Pathophysiology of Tourette syndrome (TS) and other tic disorders (TD): Cortical and cortico-subcortical hyper-excitability; Aberrant activity in the basal ganglia and abnormal basal ganglia-cortex interplay through impaired cortico-striato-thalamocortical loops; Deficient limbic cortico-subcortical loops: Altered brain monoaminergic neuromodulation.

2. Psychiatric comorbidities in TS and TD: Obsessive-compulsive disorder, attention-deficit/ hyperactivity disorder, depression, anxiety, etc. and their related neurobiological mechanisms.

3. Medication

4. Age and gender

5. Tic severity, the waxing and waning course and symptom profile

6. Stress-sensitivity substantial neurobehavioral deficits, thus aggravating neuropsychiatric symptoms and hampering cognitive and psychological development $[21,22]$. However, this issue is too often neglected in sleep research on TS/TD. Thus, age and gender across the lifespan in neurodevelopmental disorders appear to be significant factors that can affect sleep in TS/TD.

3. Since altered monoaminergic neuromodulation in TS/TD has been indicated [6], it might not be limited functionally to the motor circuits in question. According to the reciprocal interaction mesopontine brainstem system [31] (Fig. 1. upper panel), deviations in the monoaminergic/ acetylcholinergic ratio can be expected. These deviations can produce various changes in NREM and REM sleep.

4. REM sleep in TD/TS may be signified by movementrelated arousals and/or REM sleep behavioral disorder (RBD), possibly resulting from striatal/cortico-striatal dopamine overactivity $[35,36,40]$. Such links have not yet been explored.

5. 5-HT is a precursor of melatonin synthesis [31], and its decreased activity proposed in TS/TD and ADHD/OCD comorbidities $[6,14,22]$ may lead to delayed sleep onset. Indeed, difficulty in initiating sleep is a common feature in TS/TD [7-9]. However, its nature has not been adequately illuminated.

\section{Sleep Problems in TS and TD: an Overview of the Existing Literature}

In children and adolescents with TS/TD, clinical complaints of sleep problems are reported in about $25-30 \%$ of patients, with this rate being increased up to $65 \%$ when TS/TD is comorbidly diagnosed with common developmental psychiatric disorders [7, 8]. Specifically, the combination of TS and ADHD seems to carry a very high risk of sleep problems. For example, this group of patients presents as many sleep problems as TS patients, with a burden of five other comorbidities $[10,41]$. The latter, as well as cultural influences [42], makes it difficult to decide if clinically observed sleep problems in TS/ 
TD reflect a core symptom of the disorder or are merely a secondary reactive issue of clinical relevance. Further, within the context of TS/TD-associated anxiety and depression, it has to be noted that "sleep problems both predict and are predicted by a diagnostic cluster that includes oppositional defiant disorder, general anxiety disorder and depression" [43]. Sleep problems include difficulties in falling and staying asleep, separation anxiety in the evening, parasomnias (sleep walking and pavor nocturnus), and the presence of tics and other movements during sleep [7-9]. Our Medline search (up until June 2014) showed 104 publications using the key words "sleep" and "Tourette syndrome", 132 publications using the key words "sleep" and "tic disorders", 982 publications using the key words "sleep" and "ADHD", 298 publications using the key words "sleep" and "OCD", 13,844 publications using the key words "sleep" and "depression", and 8,782 publications using the key words "sleep" and "anxiety". These results clearly show that sleep problems in psychiatric comorbidities in TS/TD receive much greater attention than those in TS/TD. Notably, of the 104 publications concerning sleep in TS, only 20 original contributions and five review articles (24\%) actually targeted sleep problems in TS, and only one PSG study investigating the effect of subtle sleep alterations on daytime neurobehavioral functioning in 6- to 16-year-old children diagnosed with TS according to the Diagnostic and Statistical Manual of Mental Disorders, Third Edition, Revised has been published during the last 2 years [44••]. Similarly, only 22 original contributions and six reviews of the 132 publications concerning sleep problems in TD $(21 \%)$ have targeted the topic, with the latest studies being published in the year 2007 $[36,45,46]$. The rest of the publications that were found dealt with the effects of psychiatric comorbidities, medication, and genetic and environmental factors on sleep, rather than the actual sleep disturbances and alterations in patients with TS/ TD. Collectively, the above results indicate that essential sleep problems in TS/TD are still very insufficiently studied, with their nature being elusive.

Motor Phenomena During Sleep in TS and TD: Why Do Tics Occur Throughout Sleep, and What Is the Clinical

Significance of this Phenomenon?

Most PSG studies in children and young adults with TS/TD have shown various types of movements throughout both NREM and REM sleep, including simple and complex tics $[35,47-50]$, movements and movement-related arousals [36, $40,44 \bullet \bullet, 45,50]$, and PLMS [44••, 51]. Only two studies provided direct evidence of the presence of tics during sleep, using video monitoring $[35,48]$, while the others relied on PSG electromyographic parameters [47, 49, 50]. The issues of whether the movements and movement-related arousals found in other PSG studies [36, 40, 44••, 45] may be related to tic occurrence, or whether their presence in REM sleep may be associated with RBD in children with TS/TD, require further video monitoring verification. High prevalence of PLMS in children and adults with TS, found in only two studies [44••, 51], was not confirmed by the other PSG studies [35, 36, 40, $45,47-50]$. Thus, the high prevalence of PLMS may be due to factors unrelated to TS/TD psychopathology.

In sum, the above findings strongly indicate that the occurrence of tics during sleep in TS/TD patients is very likely. However, the actual nature and clinical significance of their presence during sleep are still elusive. We address the unresolved questions by providing several hypotheses.

1. According to recent hypotheses [17], confirmed by functional neuroimaging data $[18 \bullet]$, pathological neuronal signals from misguided basal ganglia are transmitted to premotor cortices through aberrant thalamocortical activation, thus producing sensory urges to move and the occurrence of tics. During wakefulness, a compensation originating from the PFC adaptively modulates the misguided striatal and thalamocortical oscillations. Repeated activation of these frontal systems can lead to tic suppression and deliberate alteration of the character of the movements that are involved $[17,18 \bullet$. However, during both NREM/SWS and REM sleep, the activation of PFC involved in executive control is strongly diminished or lacking [31], which can facilitate the pathological transmission from the aberrant activity in the basal ganglia and abnormal basal ganglia-cortex interplay through impaired cortico-striato-thalamocortical loops to the cortex. This hypothesis needs to be confirmed by functional neuroimaging studies and EEG investigations (sleep spindles and slow oscillations/slow-wave activity) during sleep in patients with TS/TD. Further, this hypothesis raises some questions.

(a) Are tics more or less frequent during sleep in TS/TD patients than during their daytime activity, and what roles do stress and muscle relaxation play in this respect?

(b) Where across the distinct sleep stages do tics predominate?

Whereas the first question requires intra- and interindividual monitoring of tic frequency and possible confounders across the sleep-wake cycle, the second question has been partially addressed in two PSG studies, which demonstrated more tic occurrence during REM sleep than during NREM sleep [35, 47]. These findings can be explained on the basis that NREM/SWS is characterized by the strongest reduction of synaptic connectivity [52], while the synaptic connectivity during REM sleep exceeds it during wakefulness [53]. Also, the brain regions involved in emotion generation are most active during REM sleep [31, 54], which may characterize REM sleep-related tics by strong emotionality and, probably, 
by an insufficient inhibitory functional disconnection between the central and peripheral systems in TS/TD.

2. Given the generally recognized role of sleep in memory consolidation $[25,28]$, it cannot be excluded that pathological motor programs in the aberrant basal ganglia, which are responsible for sensory urges to move that trigger tics during the day $[17,18 \bullet]$, may be consolidated and replayed during the subsequent sleep.

3. Tic occurrence during sleep may also be mediated by dreams associated with TS/TD psychopathology, because dream production originates from consolidated episodic and emotional memory sources [55].

The clinical significance of tic occurrence throughout sleep in TS/TD patients may be greater than anticipated. Tic occurrence during sleep may lead to partial sleep loss and, as mentioned above [28], insufficient and disturbed sleep results in many negative cognitive and psychological consequences which, in turn, may aggravate symptoms (tics as well as comorbidities) and impair neurobehavioral functioning. This may have a serious negative impact on social interaction, emotional status, and cognitive development, especially in a spectrum of associated developmental neuropsychiatric disorders $[21,22]$. Therefore, TS/TD mandate more intensive treatment than they have received up until now, including cognitive behavioral therapy, behavioral sleep intervention, and pharmacotherapy when necessary.

Unfortunately, in the clinical practice of TS/TD assessment, the sleep situation is often insufficiently explored. Other symptomatology overshadows this aspect, and its importance as a moderator within the dynamic reciprocal action of tics and associated psychopathology seems to be neglected. Education of physicians and patients seems to be urgently needed in order to arrive at a more complete and therapeutically relevant psychopathological picture of TS/TD.

\section{Changes in Sleep Architecture in TS and TD}

Results from several PSG studies of sleep architecture in TS/ TD patients have been relatively mixed rather than consistent. The earliest studies demonstrated decreased SWS [56], increased SWS, decreased REM sleep, paroxysmal EEG events in SWS, and frequent nocturnal arousals [48], or delayed sleep onset, fragmented sleep due to an increased number of nocturnal movements and awakenings, shortened REM sleep latency, and decreased REM sleep [49]. It is to be noted, however, that those studies used small and heterogeneous samples with respect to age, gender, coexistent comorbidities, and medication samples. Using greater and nonmedicated sample sizes, precisely matched for age, gender, and intelligence, with psychiatric comorbidities being excluded, more recent studies showed more consistent yet still relatively mixed PSG findings in patients with TS/TD. A study in young adults with TS showed decreases in sleep efficiency and the SWS percentage, and increases in sleep latency, the percentage of stage 1 of NREM sleep, the percentage of wakefulness, the number of awakenings, and sleep stage changes, while no REM sleep changes were found [35]. Another study in children with TS also found no REM sleep changes and demonstrated a longer sleep time, longer sleep latency, reduced sleep efficiency, and prolonged wakefulness after sleep onset, and less stage 2 NREM sleep and SWS [50]. Further studies in children with TS and/or TD have shown either increased REM sleep [36] or no changes in REM sleep [45], and delayed sleep onset, decreased sleep efficiency, and longer latency to SWS, as well as reduced SWS $[36,45]$. It has been suggested that increased REM sleep found in children with TS/TD might have resulted from the subclinical presence of ADHD comorbidity [36]. Yet a subsequent study demonstrated the greatest increase in REM sleep and shortened REM sleep latency in an additive fashion when ADHD and TS/TD coexisted, thus pointing to factors associated with the neurochemical mechanisms of TS/TD [46]. The latest study conducted in children and adolescents with TS found no changes in their sleep pattern but demonstrated a positive correlation between arousals from SWS and daytime expression of symptoms of conduct disorder and delinquent behavior [44*0]. It remains open as to what effect is related to TS/TD pathophysiology and/or developmental factors, since development of neuronal inhibition in TS/TD is closely related to a decrease in tics from childhood to adolescence [57].

\section{Conclusions}

Sleep research in TS/TD is still scarce relative to sleep research in other neurodevelopmental psychiatric disorders. The actual sleep problems in TS/TD patients are insufficiently studied, and their nature is elusive. This underestimation is probably due to the fact that the evidence base on etiology, assessment, and treatment is still limited $[4,6]$. The high prevalence rate of psychiatric comorbidities in TS/TD makes it even more difficult to conduct methodologically precise sleep studies. Along with the many methodological confounders listed above, lack of consideration that tics wax and wane, and that they change their typology in the course of the disorder and across the lifespan, might have produced inconsistent sleep findings.

With these accounts, we propose a multifactorial model of sleep disturbances in TS/TD, according to which a combination of factors - including TS/TD pathophysiology, psychiatric comorbidity, medication, age, gender, and tic severity (the waxing and waning course, symptom profile, and stress sensitivity) - may play a role (Fig. 1). Also, we propose future 
trends in research to more productively investigate sleep problems and their nature in TS/TD.

\section{Future Trends in Research}

1. There is still a lack of objective longitudinal sleep studies from childhood to adulthood, and such studies are necessary.

2. Targeting links between nocturnal and daily brain activation patterns in TS/TD by application of EEG and neuroimaging methodologies, in association with research on symptom expression and neurobehavioral functioning, is critically important.

3. Combining sleep and behavioral studies in TS/TD with/without ADHD, with precise phenotyping and with genetic screening, would more productively advance research in the field.

4. Further pharmacological precision of sleep and behavioral research, in combination with research on the effects of behavioral therapy, is also needed.

Finally, precise and complete clinical descriptions (by patients, parents, and experts) of sleep behavior (including movements), and its clinical relevance within the context of this complex developmental disorder, needs further clarification.

Acknowledgments The authors apologize to those whose work has not been cited in this review because of space limitations.

\section{Compliance with Ethics Guidelines}

Conflict of Interest Roumen Kirov, Andreas Becker, and Aribert Rothenberger declare that they have no conflict of interest.

Human and Animal Rights and Informed Consent This article does not contain any studies with human or animal subjects performed by any of the authors.

\section{References}

Papers of particular interest, published recently, have been highlighted as:

- Of importance

-. Of major impotance

1. American Psychiatric Association. Diagnostic and statistical manual of mental disorders: fourth edition. Text revision: DSM-IV-TR. Washington, DC: American Psychiatric Association; 2000

2. American Psychiatric Association. Diagnostic and statistical manual of mental disorders: fifth edition. DSM-5. Washington DC: American Psychiatric Association; 2013.
3. Cath DC, Hedderly T, Ludolph AG, Stern JS, Murphy T, Hartmann $\mathrm{A}$, et al. European clinical guidelines for Tourette syndrome and other tic disorders: part I. Assessment. Eur Child Adolesc Psychiatr. 2011;20:155-71.

4. Roessner V, Rothenberger A, Rickards H, Hoekstra PJ. European clinical guidelines for Tourette syndrome and other tic disorders. Eur Child Adolesc Psychiatry. 2011;20:153-4.

5. Robertson MM. The prevalence and epidemiology of Gilles de la Tourette syndrome: part 1 . The epidemiological and prevalence studies. J Psychosom Res. 2008;65:461-72.

6. Swain JE, Scahill L, Lombroso PJ, King RA, Leckman JF. Tourette syndrome and tic disorders: a decade of progress. J Am Acad Child Adolesc Psychiatry. 2007;46:947-68.

7. Freeman RD, Fast DK, Burd L, Kerbeshian J, Robertson MM, Sandor P. An international perspective on Tourette syndrome: selected findings from 3,500 individuals in 22 countries. Dev Med Child Neurol. 2000;42:436-47.

8. Freeman RD. Tourette Syndrome International Database Consortium. Tic disorders and ADHD: answers from a world-wide clinical dataset on Tourette syndrome. Eur Child Adolesc Psychiatry. 2007;16:15-23.

9. Rothenberger A, Kostanecka T, Kinkelbur J, Cohrs S, Woerner W, Hajak G. Sleep and Tourette syndrome. Adv Neurol. 2001;85:245-59.

10. Rothenberger A, Roessner V. The phenomenology of attentiondeficit/hyperactivity disorder in Tourette syndrome. In: Martino D, Leckman JF, editors. Tourette syndrome. New York: Oxford University Press; 2013. p. 26-49.

11. Ferao YA, de Alvarenga PG, Hounie AG, de Mathis MA, de Rosario MC, Miguel EC. The phenomenology of obsessive-compulsive symptoms in Tourette syndrome. In: Martino D, Leckman JF, editors. Tourette syndrome. New York: Oxford University Press; 2013. p. 50-73.

12. Robertson MM. Mood disorders and Gilles de la Tourette's syndrome: an update on prevalence, etiology, comorbidity, clinical associations, and implications. J Psychosom Res. 2006;61:349-58.

13.• Leckman JF, Bloch MH, Sukhodolski DG, Scahill L, King RA. Phenomenology of tics and sensory urges: the self under siege. In: In: Martino D, Leckman JF, editors. Tourette syndrome. New York: Oxford University Press; 2013. p. pp. 3-25. A contemporary update of the great clinical and social importance of Tourette syndrome and tic disorders.

14. Grados MA. The genetics of obsessive-compulsive disorder and Tourette's syndrome: what are the common factors? Curr Psychiatry Rep. 2009;11:162-6.

15. Leckman JF. Tourette's syndrome. Lancet. 2002;360:1577-86.

16. Moll GH, Heinrich H, Trott GE, Wirth S, Bock N, Rothenberger A. Children with comorbid attention-deficit-hyperactivity disorder and tic disorder: evidence for additive inhibitory deficits within the motor system. Ann Neurol. 2001;49:393-6.

17. Leckman JF, Vaccarino FM, Kalanithi PS, Rothenberger A. Annotation: Tourette syndrome. A relentless drumbeat - driven by misguided brain oscillations. J Child Psychol Psychiatry. 2006;47:537-50.

18. Ganos C, Roessner V, Münchau A. The functional anatomy of Gilles de la Tourette syndrome. Neurosci Biobehav Rev. 2013;37: 1050-62. Comprehensively updates neuroimaging studies of the mechanisms underpinning Tourette syndrome and tic disorders.

19. Roessner V, Plessen KJ, Rothenberger A, Ludolph AG, Rizzo R, Skov L, et al. European clinical guidelines for Tourette syndrome and other tic disorders: part II. Pharmacological treatment. Eur Child Adolesc Psychiatry. 2011;20:173-96.

20. Roessner V, Schoenefeld K, Buse J, Bender S, Ehrlich S, Münchau A. Pharmacological treatment of tic disorders and Tourette syndrome. Neuropharmacology. 2013;68:143-9.

21. Brand S, Kirov R. Sleep and its importance in adolescence and in common adolescent somatic and psychiatric conditions. Int J Gen Med. 2011;4:425-42. 
22. Kirov R, Brand S. Sleep problems and their effect in ADHD. Expert Rev Neurother. 2014;14:287-99.

23. Cortese S, Holtmann M, Banaschewski T, Buitelaar J, Coghill D, Danckaerts M, et al. Practitioner review: current best practice in the management of adverse events during treatment with ADHD medications in children and adolescents. J Child Psychol Psychiatry. 2013;54:227-46.

24. Cirelli C, Tononi G. Is sleep essential? PLoS Biol. 2008;6:e216.

25. Diekelmann S, Born J. The memory function of sleep. Nat Rev Neurosci. 2010;11:114-26.

26. Stickgold R, Walker MP. Sleep-dependent memory triage: evolving generalization through selective processing. Nat Neurosci. 2013;16: $139-45$.

27. Goldstein AN, Walker MP. The role of sleep in emotional brain function. Annu Rev Clin Psychol. 2014;10:679-708.

28. Kirov R, Brand S. The memory, cognitive and psychological functions of sleep: update from electroencephalographic and neuroimaging studies. In: Bright $\mathrm{P}$, editor. Neuroimaging - cognitive and clinical neuroscience. Rijeka: InTech; 2012. p. 155-80.

29. Rechtschaffen A, Kales A. A manual of standardized terminology techniques and scoring system for sleep stages in human subjects. US Department of Health, Education and Welfare, Public Health Service. Washington, DC: US Government Printing Office; 1968.

30. Iber C, Ancoli-Israel S, Cheeson Jr AL, Quan SF. The AASM manual for the scoring of sleep and associated events. American Academy of Sleep Medicine: Westchester; 2007.

31. Pace-Schott EF, Hobson JA. The neurobiology of sleep: genetics, cellular physiology and subcortical networks. Nat Rev Neurosci. 2002;3:591-605.

32. Li J, Hu Z, de Lecea L. The hypocretins/orexins: integrators of multiple physiological functions. Br J Pharmacol. 2014;171:33250 .

33. Hrabovszky E, Molnár CS, Borsay BÁ, Gergely P, Herczeg L, Liposits Z. Orexinergic input to dopaminergic neurons of the human ventral tegmental area. PLoS One. 2013;8:e83029.

34. Monti JM, Monti D. The involvement of dopamine in the modulation of sleep and waking. Sleep Med Rev. 2007;11:113-33.

35. Cohrs S, Rasch T, Altmeyer S, Kinkelbur J, Kostanecka T, Rothenberger A, et al. Decreased sleep quality and increased sleep related movements in patients with Tourette's syndrome. J Neurol Neurosurg Psychiatry. 2001;70:192-7.

36. Kirov R, Kinkelbur J, Banaschewski T, Rothenberger A. Sleep patterns in children with attention-deficit/hyperactivity disorder, tic disorder, and comorbidity. J Child Psychol Psychiatry. 2007;48:561-70.

37. Roffwarg HP, Muzio JN, Dement WC. Ontogenetic development of the human sleep-dream cycle. Science. 1966;152:604-19.

38. Kurth S, Ringli M, Geiger A, LeBourgeois M, Jenni OG, Huber R. Mapping of cortical activity in the first two decades of life: a highdensity sleep electroencephalogram study. J Neurosci. 2010;30: 13211-9.

39. Feinberg I, Campbell IG. Sleep EEG changes during adolescence: an index of a fundamental brain reorganization. Brain Cogn. 2010;72:56-65.

40. Trajanovic NN, Voloh I, Shapiro CM, Sandor P. REM sleep behaviour disorder in a child with Tourette's syndrome. Can J Neurol Sci. 2004;31:572-5.
41. Roessner V, Rothenberger A. Pharmacological treatment of tics. In: Martino D, Leckman JF, editors. Tourette syndrome. New York: Oxford University Press; 2013. p. 524-52.

42. Rothenberger A, Becker A, Rothenberger LG. Core features and associated psychopathology of Tourette syndrome: modifications by cultural aspects? Eur Child and Adolesc Psychiatry. 2013;22: 709-11.

43. Shanahan L, Copperland WE, Angold A, Bondy CL, Costello E. Sleep problems predict and are predicted by generalized anxiety/ depression and oppositional defiant disorder. J Child Adolesc Psychiatry. 2014;53:550-8.

44.• Stephens RJ, Chung SA, Jovanovic D, Guerra R, Stephens B, Sandor P, et al. Relationship between polysomnographic sleep architecture and behavior in medication-free children with TS, ADHD, TS and ADHD, and controls. J Dev Behav Pediatr. 2013;34:688-96. Investigates relationships between sleep alterations and daytime neurobehavioral functioning in youth with Tourette syndrome for the first time.

45. Kirov R, Roessner V, Uebel H, Banaschewski T, Kinkelbur J, Rothenberger A. Sleep behavior in children with tic disorders-a polysomnographic study [in German]. Z Kinder Jugendpsychiatr Psychother. 2007;35:119-26.

46. Kirov R, Banaschewski T, Uebel H, Kinkelbur J, Rothenberger A. REM-sleep alterations in children with co-existence of tic disorders and attention-deficit/hyperactivity disorder: impact of hypermotor symptoms. Eur Child Adolesc Psychiatry. 2007;16:45-50.

47. Hashimoto T, Endo S, Fukuda K, Hiura K, Kawano N, Suzue J, et al. Increased body movements during sleep in Gilles de la Tourette syndrome. Brain Dev. 1981;3:31-5.

48. Glaze DG, Frost Jr JD, Jankovic J. Sleep in Gilles de la Tourette's syndrome: disorder of arousal. Neurology. 1983;33:586-92.

49. Drake Jr ME, Hietter SA, Bogner JE, Andrews JM. Cassette EEG sleep recordings in Gilles de la Tourette syndrome. Clin Electroencephalogr. 1992;23:142-6.

50. Kostanecka-Endress T, Banaschewski T, Kinkelbur J, Wüllner I, Lichtblau S, Cohrs S, et al. Disturbed sleep in children with Tourette syndrome: a polysomnographic study. J Psychosom Res. 2003;55: 23-9.

51. Voderholzer U, Müller N, Haag C, Riemann D, Straube A. Periodic limb movements during sleep are a frequent finding in patients with Gilles de la Tourette's syndrome. J Neurol. 1997;244:521-6.

52. Massimini M, Ferrarelli F, Huber R, Esser SK, Singh H, Tononi G. Breakdown of cortical effective connectivity during sleep. Science. 2005;309:2228-32.

53. Massimini M, Ferrarelli F, Murphy M, Huber R, Riedner B, Casarotto $\mathrm{S}$, et al. Cortical reactivity and effective connectivity during REM sleep in humans. Cogn Neurosci. 2010;1:176-83.

54. Kirov R, Brand S, Kolev V, Yordanova J. The sleeping brain and the neural basis of emotions. Behav Brain Sci. 2012;35:155-6.

55. Llewellyn S. Such stuff as dreams are made on? Elaborative encoding, the ancient art of memory, and the hippocampus. Behav Brain Sci. 2013;36:589-607.

56. Mendelson WB, Caine ED, Goyer P, Ebert M, Gillin JC. Sleep in Gilles de la Tourette syndrome. Biol Psychiatry. 1980;15:339-43.

57. Moll GH, Heinrich H, Gevensleben H, Rothenberger A. Tic distribution and inhibitory processes in the sensorimotor circuit during adolescence: a cross-sectional TMS study. Neurosci Lett. 2006;403:96-9. 